\title{
Using UAVs for Remote Study of ice in the Arctic with a View to Laying the Optimal Route Vessel
}

\author{
Alexey Lagunov \\ Northern (Arctic) Federal University \\ named after M. V. Lomonosov \\ ul. Severnaya Dvina Emb. 17, \\ Arkhangelsk, Russia \\ E-mail: a.lagunov@narfu.ru
}

\author{
Dmitry Fedin \\ Northern (Arctic) Federal University \\ named after M. V. Lomonosov \\ ul. Severnaya Dvina Emb. 17, \\ Arkhangelsk, Russia \\ E-mail: d.fedin@narfu.ru
}

\author{
Anatoliy Tyagunin \\ Northern (Arctic) Federal University \\ named after M. V. Lomonosov \\ ul. Severnaya Dvina Emb. 17, \\ Arkhangelsk, Russia \\ E-mail: av.tyagunin@yandex.ru
}

\begin{abstract}
Arctic Development is one of the strategic goals of Russia and other circumpolar countries. Solution of this problem is faced with the problem: most of the coastal seas and the Arctic Ocean is covered with ice. For large vessels and icebreakers problem is solved by applying remote sensing of ice with spacecraft and aircraft. This solution has a very high cost and low efficiency. The authors propose to use for this purpose unmanned aerial vehicles (UAVs). This paper describes the solution of choice of UAV. This paper describes the solution of choice UAV. The features of the use of equipment for photo and video and microwave sensing of ice.
\end{abstract}

\section{INTRODUCTION}

$\mathrm{T}$ HE Arctic is extremely important military-strategic significance for Russia. Mission of Northern (Arctic) Federal University named after M.V. Lomonosov - formation and development of competitive human capital in the North-West Federal District through the creation and implementation of innovative services, development from the perspectives of the development of the Russian North and the Arctic. To achieve this mission NArFU has highly skilled specialists, high-tech equipment, the ability to introduce new developments in the production and achieve the desired result. Russian Government commissioned a comprehensive research in the Arctic is the NArFU

Arctic exploration is largely hampered by the presence of ice in the Arctic Ocean and adjacent seas. The problem of predicting the state of the ice: its thickness, density, size of the ice fields - is one of the most important issues of today. Methods of direct examination by the state of the ice landing on the surface is not always applicable for reasons of safety. For forecasting ice conditions are more commonly used methods of distance study.

Remote sensing - sensing the Earth's surface is on board aircraft or equipment from space using the properties of electromagnetic waves emitted, reflected or scattered by the sensed objects, for the purpose of providing information for disaster management, improve management of natural resources land use management and environmental protection.
Remote Sensing - a set of methods for study of the atmosphere, the earth's surface, the oceans, the top layer of the earth's crust by air aerospace methods based on the decoding of images obtained at a distance from the aircraft.

The result is:

1. Aerial photographs obtained from a height of preferably $500 \mathrm{~m}$ up to $10 \mathrm{~km}$, but not more than $30 \mathrm{~km}$;

2. Space images - from a height of $150 \mathrm{~km}$.

Operating regions for the filming freeze commonly use the following satellites:

1. The radar KA RADARSAT-1 and ENVISAT-1 to provide a guaranteed all-weather shooting at any time of day.

2.Highly optical satellites EROS A/B for a detailed analysis of regions ice and flooding, SPOT multispectral optical unit 4 for the analysis of ice on rivers, ice crossings and in areas of potential ice blocks.

All remote sensing techniques for observing the Earth's surface use electromagnetic (EM) radiation in the visible/nearinfrared (VNIR), thermal infrared (TIR) or microwave bands of the electromagnetic spectrum. There are three principal measurement techniques applicable to ocean and sea ice observations:

1. Measurement of the part of the incoming solar radiation that is reflected at the surface of the Earth (visual and near-infrared remote sensing).

2. Measurement of the thermal radiation from the surface (thermal infrared and passive microwave remote sensing).

3. Measurement of the return signal from an active source, such as a microwave radar, using several types of instruments that measure backscattered radiation from the surface.

The sea ice surface can be considered to have three macroscopic components:

1. Open water in leads, polynyas and melt ponds on top of the ice in summer.

2. Ice with varying amounts of salt water intrusions (salt pockets).

3. Snow on top of the ice. 
The following properties of the macroscopic components have impact on the remote sensing measurements:

1. Percentage and distribution of the three components.

2. Temperature of the components.

3. Salinity of the components and the distribution of salt intrusions in the ice.

4. Crystal structure of the ice and the snow.

5. Occurrence of snow and ice layers, and rough surfaces (e.g., floes, ridges, frost flowers).

In addition, there are variables defined by the remote sensing instrument that also have significant impact on the measurements:

1. Frequency (wavelength) of the radiation.

2. Angle of incidence of the radiation.

3. Polarization of the radiation if the view is not from directly above.

The main disadvantages of the methods at work in the Arctic:

1. The use of space or air assets has a high cost, so it can be used only for large vessels (nuclear icebreakers, military ships, tankers). For smaller vessels, which often carry out scientific research and commercial activities (fishing, harvesting of marine animals, environmental activities) use of these funds because of high prices is impossible.

2. Low levels of efficiency: for taking pictures requires considerable time. Receive data in real time is difficult.

3Files aerial and satellite images are quite large, making it difficult to transmit this information via communication channels. In the Arctic, there are problems with communication, mainly by satellite, which is unsustainable. This leads to the fact that the communication channels are quite narrow and has a high data transfer rates [1].

We propose to explore the question of the use of unmanned aerial vehicles (UAV).

\section{II.UNMANNED AERIAL VEHICLES}

Stimulus to the development of unmanned aircraft in the world was the successful and widespread use of UAVs by the armies of the United States and Israel during the military operations (Persian Gulf, Yugoslavia, the Middle East, the Arab-Israeli wars). While drones have proven to be an effective means of intelligence, combat support, as false targets for the detection of enemy air defense systems, delivery of goods, to perform other missions.

UAV - in general, this aircraft without crew on board. The notion of an aircraft includes a large number of types, each of which has its analogue drone. In this article the definition of UAV misses the following concept: aircraft without crew on board, using the principle of creating aerodynamic lift by fixed or rotary wing (UAV aircraft and helicopter type), equipped with an engine and having a payload and flight duration, sufficient to perform specific tasks.
One of the major problems with UAVs - management of the unit.

There are the following control methods:

1. Manual control of the operator (or remote piloting) with remote control within optical observability or specific information from the forward-looking video camera. This control operator primarily solves the problem of piloting: maintaining a desired course, altitude, etc.

2. Automatic control provides the ability to fully autonomous UAV flight along a predetermined path at a predetermined height at a given rate and the stabilization of the orientation angles. Automatic operation via onboard software devices.

3. Semi-automatic control (or remote control) - flight is performed automatically without human intervention using the autopilot initially set parameters, but the operator can make changes to the route online. Thus, the operator has the ability to influence the outcome of the operation that is focused on the task of piloting.

Manual control can be one of the modes for the UAV, and may be the only way to manage. UAVs, deprived of any means of automatic flight control.

Last two methods are currently the most popular among users of unmanned systems, as impose the least training requirements and ensure safe and efficient operation of UAV systems Fully automatic control can be an optimal solution for a given section of aerial tasks when you need to shoot at a great distance from home base is in contact with the ground station. At the same time, as the responsible person for the flight, the launching, the opportunity to influence the flight from the ground station can help avoid emergency situations.

To perform specific tasks, particularly for aerial photography and remote sensing, UAV should be considered in conjunction with its instrumentation and payload, which introduced the term unmanned aircraft system (UAS).

UAS, in addition to the UAV consists of onboard control complex, payload and ground control station.

1. Onboard complex:

- Integrated Navigation System;

- Satellite Navigation System Receiver;

- Autopilot. Autopilot problem:

a) Piloting: automatic flight on a given route, automatic takeoff and landing approach, maintaining the desired altitude and airspeed, the stabilization of the orientation angles, forced landing in the event of engine failure or other serious problems.

b) Software control onboard systems and payloads, such as camcorder stabilization and synchronization time and coordinates of the camera shutter, release the parachute.

- Drive flight information. 
2. To payload for remote studies of ice refers digital camera, can be used as addition video camera, thermal imager, infrared camera, microwave complex.

3. Ground control functions: Flight tracking, Data receiving, Transfer commands.

Formulate a series of signs to determine the UAV used for the remote research of ice:

1. Type: UAV aircraft or helicopter type.

2. Control mode: automatic or semi-automatic.

3. UAV remote research of ice must have on board a complete autopilot, capable of withstanding the shooting parameters (route angles equipment, the percentage of longitudinal and transverse floors, height, etc.) even at low weight machine in a wide range of weather conditions.

4. Payload: calibrated automatic digital camera or microwave module (possibly as an adjunct video camera, a thermal imager and infrared camera), no excessive payload needed for military drones.

5. For today it should be models flying at low altitudes (in the class $\mathrm{G}$ airspace with a height of up to $4.5 \mathrm{~km}$ in unpopulated areas, within which it is planned to introduce a notification procedure for small and fly unmanned aircraft). Obtaining permission to fly in Class $\mathrm{A}$ and $\mathrm{C}$ while it is possible only by the military.

4. Commercially available - stood the test flights and entered production.

5. The model performed photogrammetric projects that have links on the site, or based on projects released article. The company's website is an indication that the main or one of the purposes is aerial photography.

\section{MICROCOPTER}

In 2006, the German enthusiasts Holger Buss and Ingo Busker created a microcopter (MC) around which gathered a large community of enthusiastic people - modelers, designers and programmers. Already in mid-2007, through the efforts of the founders and the entire community of this project $\mathrm{MC}$ has hovered steadily and quickly moved through the air. Microcopter is a radio-controlled flying platform with $4,6,8,12$ brushless motors with propellers. In flight platform takes horizontal position relative to the earth's surface, can hover over a certain place, move left, right, forward, backward, up and down. Nowadays, thanks to the additional equipment developed microcopter has the opportunity to actually perform semi-autonomous flight.

Along with other quite successfully implemented similar projects $\mathrm{MC}$ project has a very extensive support that will be needed and the novice and the experienced modeler that uses all the features of this unit.

MC flight can be considered an example hexacopter (option unit with 6 motors), sets with different number of motors are controlled in the same way. Hexacopter has propeller pairs which rotate in the opposite direction. Machine Diagram is shown in Fig.1.

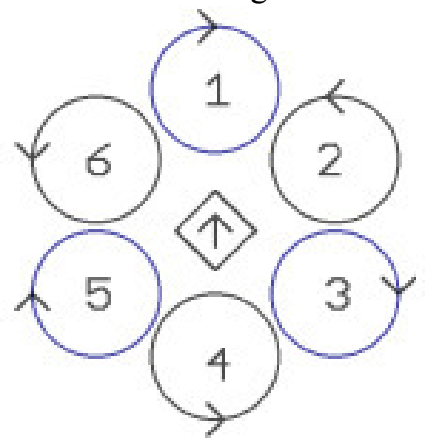

Fig. 1. Schematic rotation of hexacopter propellers.

As shown in the diagram, 1, 3, 5 hexacopter propellers rotate clockwise and 2. 4, 6 counterclockwise. To soar, all propellers must rotate at the same speed. Since the same number of propellers rotate in opposite directions at the same time, the force between them is equalized and allows to the hexacopter stably float in the air, not rotating around its axis. Photo of the machine shown in Fig.2.

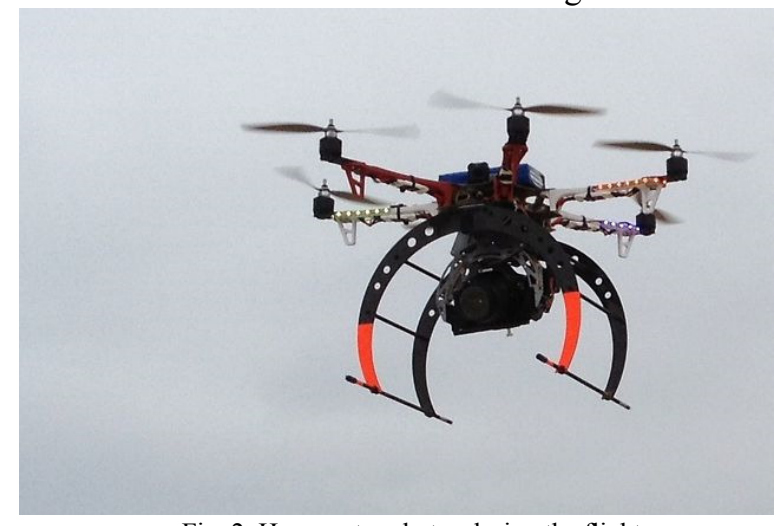

Fig. 2. Hexacopter photos during the flight.

To fly in any direction, hexacopter should come out of balance, which increases the rotational speed of the propeller, opposite the direction of movement required. It makes hexacopter bend and move in this direction. For example: to fly forward, rear propeller must spin faster ("nick"). The word "nick" is used as the name for the movement of the machine forward or backward. Left or right movement called "rolls". Turn on the spot called "yaw". To implement "yaw", need to upset the balance of speed between the front / rear and left / right propellers: to turn the unit around its axis in a clockwise direction, front / rear propeller must rotate faster, and left / right propellers bit slow. Thus, carried hexacopter turn clockwise keeping the height at which the unit is located.

To ensure sustained flight controller is required which main task is to manage the flying platform stabilization in the air in a horizontal position by supplying control signals engines. It uses data from multiple sensors and calculates a speed for each propeller. The controller also compensates for external influences, such as wind. The controller works in a special program. 
Key design MC elements: Baseboard (FlightCtrl), Brushless motor controllers (BL-Ctrl), Brushless motors (BLmotors), Chassis, LiPo battery, Propellers, Radioreceiver, Transmitter, Connecting wires, connectors.

Additionally microcopter can be equipped with the following elements:

1. Height sensor (barometer) - with the help of the device acquires the ability of flight at a constant altitude;

2. Magnetic compass (magnetometer) - with the help of the machine is able to hold the longitudinal axis in a constant direction;

3. GPS-receiver, which determines the coordinates of the vehicle in space and, therefore, it can provide a securing position for the given coordinates, i.e. fly on the specified points on the routes, and return to the starting point (this arrangement can also provide additional data for such telemetry as altitude, speed, direction,);

4. Sound locator (sonar), with which it is possible more precise hold altitude (it is used at an altitude of up to $6 \mathrm{~m}$ as an alternative barometer; moreover, it is used in the automatic takeoff and landing);

5. Optical sensor that allows precise positioning of the aircraft vertically to a height of $2.5 \mathrm{~m}$

To use MC also needed: Chargers for LiPo batteries, Set of tools and accessories.

Issues requiring resolution:

1. Increased flight time - in research requires more flight time, at least 45 minutes;

2. Increase in all weather and range use microcopter;

3. Automation of some operations use multicopter;

4. Improved safety - a safe landing when connection is lost, the protection of propellers, etc.

A configuration microcopter was decided to use the UAV with six rotors (Hexacopter). Choice is due to the aerodynamic scheme balanced multi rotor aircraft. Unlike quadrocopter, failure of one or two rotors can be controlled landing. Despite lower gas-dynamic efficiency due to a smaller propeller group wheelbase engines selected design is more secure.

Depending on the task many rotary UAV can:

- lifted to a height of the payload of up to $2,000 \mathrm{~m}$ (the load is fixed at the central portion of the machine, around which are located coaxially or from 3 to 12 engines);

- «hang" on a user specified height with the ability to smoothly zoom in and outперемещаться во всех направлениях с полезной нагрузкой со скоростью до

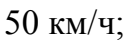

- be in flight up to 30 minutes (flight time the machine depends on its configuration and payload);

- operated in a wide range of ambient temperatures and wind speed of $20-25 \mathrm{~m} / \mathrm{s}$ (operation in rainy weather and snowfall during the difficult, but still possible);
- perform autonomous flight route specified coordinates on the navigation map, with stops at the desired points on the operator specified time;

- perform at stops aerial photography area, holding a given operator height and position using GPS coordinates;

- back to a place of take-off from any point on the route and from any position held by a signal operator;

- upon loss radio implement decrease with preprogrammed speed or to return to the starting point of a GPS;

- have a mobile structure (about the size of $30 \times 30 \times 50$ $\mathrm{cm}$ when folded), allowing to bring the machine to a working state from traveling for 1-5 minutes;

- provide guidance onboard aerial camera television system to the subject.

Unmanned complex has on board three independent radio telemetry and positioning. One of the channels displayed on the computer, the other is connected to a standalone module indicating that lie directly in the pilot. The third channel is built into the radio equipment control. Telemetry provides continuous monitoring of all critical flight parameters and operating modes of complex systems. Management also dubbed by second post connected to a personal computer via radio. It records all flight data on the memory card, which allows for the completion of the flight to make his "analysis." Onboard video transmitter broadcasting provides high quality images to a ground monitor in real time to provide accurate guidance photos and video. The complex is equipped with a gyroscopic stabilization system with camera tracking mode. It provides image stability and vibration-free even under difficult conditions, aerial photography. Video transmitted to the monitor board operator who controls the position of the camera during the aerial survey.

\section{FEATURES WITH UAV AERIAL DATA}

Aerial UAV is not fundamentally different from shooting with "large aircraft", but has certain features that we continue to consider. UAV flight, usually made with a cruising speed of 70-110 km / h (20-30 m / c) at altitudes of 300-1500 m. Usually used for shooting non-metric camera household with matrix size 10-20 megapixels. Focal length of the chamber is typically about $50 \mathrm{~mm}(35 \mathrm{~mm}$ equivalent), which corresponds to pixel size in the terrain (GSD) of from 7 to $35 \mathrm{~cm}$. Often images are processed with UAV simple lax methods (affine transformation pictures to the plane). As a result, the user receives a block layout, which, in addition to low precision circuits may contain gaps at the joints adjacent shots.

In this article, we start from a rigorous photogrammetric data processing, as a result of which we can expect the accuracy of the results (usually orthophoto mosaics) about one GSD. When the parameter values taken, the above 
results correspond to the scale accuracy orthophoto from 1:500 to 1:2000 depending on the height of the shooting.

For rigorous photogrammetric aerial data and obtain the most accurate results to images in the same route had a triple overlap, and the overlap between adjacent strips in areal recording is at least $20 \%$. In practice, when taking UAV these parameters are not always maintained. Affect the flight of the UAV gusts, turbulence, and other confounders. If shooting with conventional aircraft plan overlapped along the route $60 \%$, and between $20-30 \%$ of the routes, the design should be shot with UAV along with overlapping routes $80 \%$, and between routes - $40 \%$ [2].

To improve the quality of the images is recommended to install on the flight chamber lenses with fixed focal length. When shooting should exhibit focuses on infinity and disable the "AF".

Pictures of digital cameras, both amateur and professional, have a rectangular shape. "Advantageous" position the camera so that the long side of the image extends across the flight - it can take a large area with the same length of the route. Survey should be carried out with the highest quality - with the smallest jpeg compression or in RAW, if the latter is possible.

The present level of navigational aids allows for measurement of exterior orientation directly in the process of shooting. Typical accuracy of such measurements reach a few centimeters over the spatial coordinates $\mathrm{X}, \mathrm{Y}$ and $\mathrm{Z}$ and 0.005 degrees angles roll, pitch and yaw for the most accurate systems. Often this is enough to make processing without the use of reference points. In any case, the presence of such data greatly simplifies the handling and enables some of the processing steps in fully automatic mode. Recent advances in microelectronics allow to collect the mechanical (or rather MEMS - electro-mechanical) gyroscope package size of a few mm, costing from $\$ 250$. Such precision gyroscopes do not give a professional, have considerable care (about one degree per hour) during operation, but greatly simplify subsequent data processing. Passport accuracy GPS devices is $10 \mathrm{~mm}+1.5 \mathrm{~mm} \times \mathrm{B}$ (B removal from the base station in $\mathrm{km}$ ) horizontal and $20 \mathrm{~mm}$ $+1.5 \mathrm{~mm} \times \mathrm{B}$ adjustment. Unfortunately, usually on board the UAV mounted cheaper GPS receivers and shall not establish IMU sensors. Data centers in the projection images are shot through telemetry data NMEA protocol and have in this case, the accuracy of 20-30 m, and the angles of pitch, roll and yaw are calculated from the velocity vector of GPS measurements. Yaw angle accuracy in such telemetry data is low and can exceed 10 degrees and the values themselves contain systematic errors, which complicates the subsequent data processing.

When shooting with a dual-band GPS receiver used in differential mode (or PPP data processing GPS), then the minimum number of control points for the most accurate results processing. Usually enough points 100 1-2 shots, in some cases the treatment can be carried out without GCP. In the case where there is no accurate projection centers, the requirements for horizontal and vertical justification of the standard, one horizontal and vertical point on 6-10 shooting bases.

\section{V.MicROWAVE SENSING}

Work systems radiophysics diagnostics and control interference environments based on an analysis of the reaction medium under investigation by the probe signal.

Task adequate description of the interaction of electromagnetic waves with the probed environment characterized by complex permittivity $\left(\varepsilon^{*}\right)$, is one of the most pressing. This is due to the fact that the material of the medium of sensed are complicated dielectric structure. In reality, these media are in constant contact with a variable temperature field and the water in its various states of aggregation. These variables determine the components, mainly the dielectric properties of such media.

In solving problems of radiowave diagnostic status and properties of layered media is necessary to consider the spatial distribution of $\varepsilon^{*}$. Data on their core distribution $\varepsilon^{*}$ can be obtained either from a priori data or using approximate theoretical models or experimentally. Analyze the value of the reflection coefficient of plane waves vertical and horizontal polarization at oblique incidence on a controlled environment. Sharing the results of sensing vertically and horizontally polarized waves can extract information on the dielectric properties of sensed layers.

Of free space $(\varepsilon *=1, \mu *=1)$ on the layered dielectric medium falls flat electromagnetic wave at different angles $\Theta$ (Fig.3) [3].

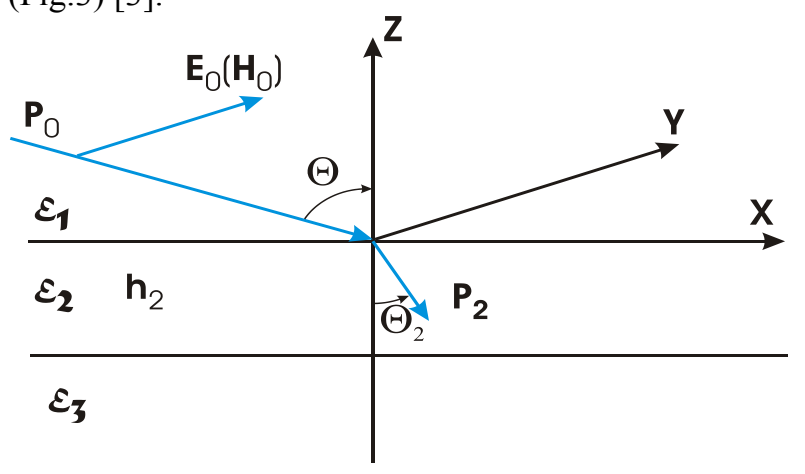

Fig.3.Geometry of the problem, the vertical and horizontal-E0-H0 polarization

Required to determine the value of the reflection coefficient $\mathrm{K}$ of the investigated medium, depending on the angle of incidence of the waves of horizontal and vertical polarization in the case of a finding on the medium surface of the dielectric layers. The upper and lower layers have a semi-infinite thickness, and the thickness of the relatively thin second layer - and the variable commensurate with the 
wavelength. In this case, the first layer - the air, the second layer - the ice, and the third - the water. $\varepsilon^{*}$ values of the second and third layer are changed during the experiment..

To carry out numerical simulation environment with specialized distribution $\varepsilon *$ can be represented as a multilayer system. In this case, $\varepsilon^{*}$ is a function of the coordinates of $\mathrm{Z}$, and at the boundaries between the layers, this function may be discontinuous. Dependence of $\varepsilon^{*}(\mathrm{Z})$ within each layer is given by the numerical values at certain points $\mathrm{Zi}$. To simplify the calculations, we set $\varepsilon *$ between points $\mathrm{Zi}$ and $\mathrm{Zi}+1$ is a constant and uniform in $\mathrm{X}$ and $\mathrm{Y}$ directions in layers.

Reflectance of a multilayer medium can be determined by the recurrence formula [1]:

$$
\begin{aligned}
& K_{1, n}=\frac{K_{1,2}+K_{2, n} e^{-j \frac{4 \pi h_{2}}{\lambda \sqrt{\varepsilon_{2}}}}}{1+K_{1,2} K_{2, n} e^{-j \frac{4 \pi h_{2}}{\lambda \sqrt{\varepsilon_{2}}}}} \\
& K_{i, i}=0, \quad K_{i, i+1}=\frac{\sqrt{\varepsilon_{i+1}}-\sqrt{\varepsilon_{i}}}{\sqrt{\varepsilon_{i+1}}+\sqrt{\varepsilon_{i}}} \\
& K_{i, k}=\frac{K_{i, i+1}+K_{i+1, k} e^{-j \frac{4 \pi h_{i+1}}{\lambda \sqrt{\varepsilon_{i+1}}}}}{1+K_{i, i+1} K_{i+1, k} e^{-j \frac{4 \pi h_{i+1}}{\lambda \sqrt{\varepsilon_{i+1}}}}} \quad k \neq i, k \neq i+1
\end{aligned}
$$

Using the formulas (1-3), we find the formula for the reflection coefficient K1-3 in the case research we have adopted the model:

$$
K_{1,3}=\frac{K_{1,2}+K_{2,3} e^{\gamma_{1}}}{1+K_{1,2} K_{2,3} e^{\gamma_{1}}}, \quad \text { əдe } \quad \gamma_{1}=-j \frac{4 \pi h_{2}}{\lambda \sqrt{\varepsilon_{2}}}
$$

then for horizontal polarization:

$K_{1,2}=\frac{\sqrt{\varepsilon_{1}} \cos \Theta-\sqrt{\varepsilon_{2}-\varepsilon_{1}(\sin \Theta)^{2}}}{\sqrt{\varepsilon_{1}} \cos \Theta+\sqrt{\varepsilon_{2}-\varepsilon_{1}(\sin \Theta)^{2}}}$

$K_{2,3}=\frac{\sqrt{\varepsilon_{2}} \cos \Theta_{2}-\sqrt{\varepsilon_{3}-\varepsilon_{2}\left(\sin \Theta_{2}\right)^{2}}}{\sqrt{\varepsilon_{2}} \cos \Theta_{2}+\sqrt{\varepsilon_{3}-\varepsilon_{2}\left(\sin \Theta_{2}\right)^{2}}}$,

где $\Theta_{2}=\arcsin \left(\frac{\sin \Theta}{\sqrt{\varepsilon_{2}}}\right)$

for vertical polarization:

$$
\begin{aligned}
& K_{1,2}=\frac{\varepsilon_{2} \cos \Theta-\sqrt{\varepsilon_{1}\left(\varepsilon_{2}-\varepsilon_{1}(\sin \Theta)^{2}\right)}}{\varepsilon_{2} \cos \Theta+\sqrt{\varepsilon_{1}\left(\varepsilon_{2}-\varepsilon_{1}(\sin \Theta)^{2}\right)}} \\
& K_{2,3}=\frac{\varepsilon_{3} \cos \Theta_{2}-\sqrt{\varepsilon_{2}\left(\varepsilon_{3}-\varepsilon_{2}\left(\sin \Theta_{2}\right)^{2}\right)}}{\varepsilon_{3} \cos \Theta_{2}+\sqrt{\varepsilon_{2}\left(\varepsilon_{3}-\varepsilon_{2}\left(\sin \Theta_{2}\right)^{2}\right)}}
\end{aligned}
$$

By (4-8) for different states of the environment were calculated modules reflection coefficients for horizontal waves $|\mathrm{KH}|$ and vertical $|\mathrm{KV}|$ polarizations at various angles of incidence $\Theta$ on sensed environment. In this varied thickness of the thin layer h2, set different values $\varepsilon *$ thin layer and the third layer. For clarity, the thickness of the thin layer was set in relative units and normalized with the wavelength in the medium

$$
\begin{gathered}
\gamma_{1}=-j \frac{4 \pi h_{2}}{\lambda \sqrt{\varepsilon_{2}}}=-j \frac{4 \pi H}{\varepsilon_{2}} \\
\text { где } H=\frac{h_{2}}{\lambda_{c p .}}, \lambda_{c p .}=\frac{\lambda}{\sqrt{\varepsilon_{2}}}
\end{gathered}
$$

We simulated the situation. One of the results for the horizontal and vertical polarization is shown in Fig.4 and Fig.5.

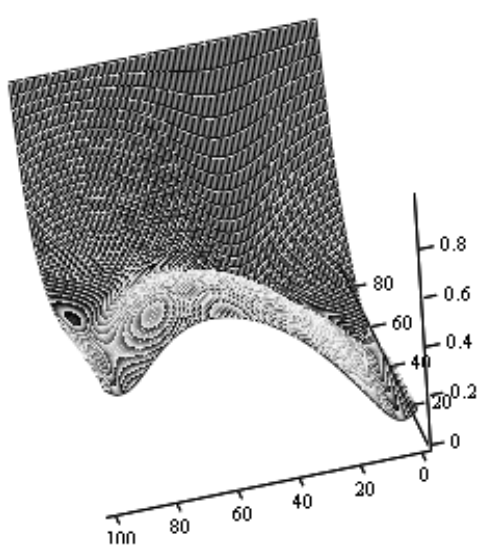

Fig. 4. Model for vertical polarization

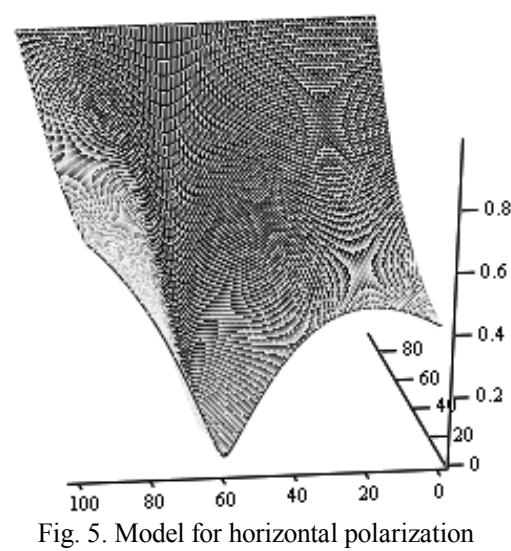

The $\mathrm{X}$ axis shows the angle $\Theta$, on the $\mathrm{Y}$-axis the thickness of the ice along the axis $Z$ - the reflection coefficient. Chart analysis shows that horizontal polarization wave we have higher reflectance. Therefore, we will use the antenna, allowing to receive horizontal polarization. For microwave module to reduce the weight of the flight apparatus we used stroboscopic method.

\section{EXPERIMENTAL RESEARCH}

Experimental study was conducted in the delta of the Northern Dvina and dry sea near the island Mudyug. 
UAV showed good performance on the stability of the enroute. Photo and video possible to determine accurately enough to crack the ice and to estimate the size of ice fields.

Microwave sensing results are shown in Table I. Analysis of the results showed that the error in determining the ice thickness is about $15 \%$.

TABLE I.

\section{EXPERIMENTAL RESULTS}

\begin{tabular}{|l|c|c|}
\hline \multicolumn{1}{|c|}{ Measurement point } & \multicolumn{2}{|c|}{ Ice thickness (m) } \\
\cline { 2 - 3 } & $\begin{array}{c}\text { Microwave } \\
\text { sensing }\end{array}$ & Drilling \\
\hline $\begin{array}{l}\text { Point number 1 r. Northern Dvina } \\
64^{\circ} 26^{\prime} 59.27^{\prime \prime} \mathrm{N} 40^{\circ} 49^{\prime} 2.7^{\prime \prime} \mathrm{E}\end{array}$ & 0,21 & 0,38 \\
\hline $\begin{array}{l}\text { Point number 2 r. Northern Dvina } \\
64^{\circ} 28^{\prime} 10.23^{\prime \prime} \mathrm{N} 40^{\circ} 48^{\prime} 42.93^{\prime \prime} \mathrm{E}\end{array}$ & 0,58 & 0,42 \\
\hline $\begin{array}{l}\text { Point number 3 r. Northern Dvina } \\
64^{\circ} 27^{\prime} 1.93^{\prime \prime} \mathrm{N} 40^{\circ} 51^{\prime} 5.07^{\prime \prime} \mathrm{E}\end{array}$ & 0,43 & 0,4 \\
\hline $\begin{array}{l}\text { Point number 1 Dry sea } \\
64^{\circ} 52^{\prime} 25.32^{\prime \prime} \mathrm{N} 40^{\circ} 18^{\prime} 10.27^{\prime \prime} \mathrm{E}\end{array}$ & 0,61 & 0,48 \\
\hline $\begin{array}{l}\text { Point number 2 Dry sea } \\
64^{\circ} 55^{\prime} 3.55^{\prime \prime} \mathrm{N} 40^{\circ} 18^{\prime} 39.94^{\prime \prime} \mathrm{E}\end{array}$ & 0,57 & 0,47 \\
\hline $\begin{array}{l}\text { Point number 3 Dry sea } \\
64^{\circ} 54^{\prime} 22.63^{\prime \prime} \mathrm{N} 40^{\circ} 23^{\prime} 8.14^{\prime \prime} \mathrm{E}\end{array}$ & 0,6 & 0,49 \\
\hline
\end{tabular}

In our data detected by sensing was carried out expeditionary ground survey of ice cover. Ground survey data confirmed the presence of breaks plots autumn ice ships that were detected on the river was probed image even in the presence of ice on the surface of the snow layer thickness of $35-40 \mathrm{~cm}$.

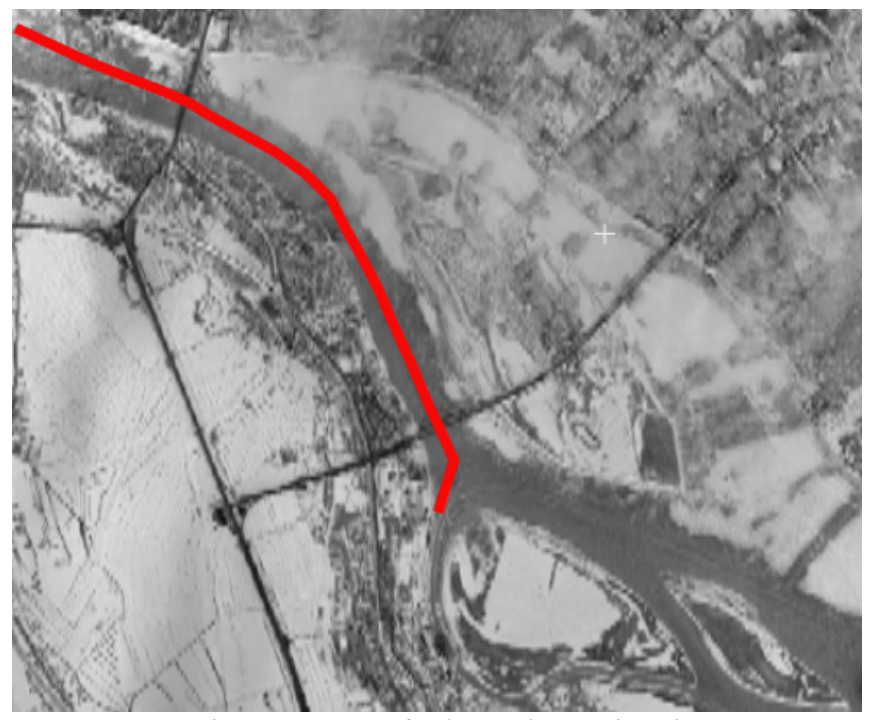

Fig. 6. Route Map for the Northern Dvina River

This happened due to the nature of hummocky surface and metamorphosed ice structure, consisting of the frozen broken ice, slush and slush interspersed with lots of ice and fine sand particles. Due to scattering of light has broken-ice has a characteristic white color, and so it is sometimes referred to as white ice (as opposed to dark crystal clear "black" - ice).This type of ice texture in contrast to dark ("black") reflecting intense electromagnetic waves in the centimeter range. The presence of intense white spots and stripes on the was probed image is an important deciphering sign of milled icebreakers of ice cover. Such assessment of ice covered with a layer of snow, it is impossible according aerorazvedki or visual ground survey, as well as methods of remote sensing in the optical wavelength range. The study traced the route on the Northern Dvina River (Fig.6).

\section{CONCLUSION}

In the study of the possibilities of remote ice research, we concluded that the application of classical methods: spacecraft and aircraft uneconomical and has low efficiency. In addition, using these tools, we are faced with the problem of transmitting data through a narrow channel.

To solve this problem, we selected unmanned aerial vehicles (UAVs), which lack the above drawbacks, but have weight limits of the equipment used. We conducted an analysis of existing UAV and concluded that the best fit for our problem microcopter.

UAV have the following advantages:

1. Low cost.

2. Small size and weight that allows you to place equipment even on small vessels.

3. Data may be transmitted from UAV quickly in real time.

4. With a small UAV flight range can be used for broadband data transmission channel. This allows you to use equipment with high resolution.

5. Can be used in disaster areas without risk to life and limb of the operator (debacle, flooding).

Disadvantages of using UAVs:

1. Low level of accuracy of the measurements.

2. The limited size of the payload.

3 . Dependence on weather conditions.

To capture an image, we propose to use a digital camera with a fixed focal length.

For remote sensing of ice, we propose to use the frequency range 1-2 $\mathrm{GHz}$ and microwave sensing stroboscopic method.

Experiments have shown the possibility of using UAVs for remote sensing of ice. Error in determining the thickness of the ice was not more than $15 \%$.

\section{REFERENCES}

[1] Anpilogov VR, AA Afonin Attenuation in the satellite channels Ku-and Ka-band [electronic resource] - Access mode: http://www.tssonline.ru/articles2/practicum/zatyhanie-v-spytnikovihkanalah-ku-i-kadiapazonov\#sthash.CRQJPZ21.dpuf. Retrieved $11 / 21 / 2013$

[2] Scoobyev SI, Research and Production Institute of Land and Information Technology of the State University of Land "Zeminform" (Russia), The use of unmanned aerial vehicles for the purposes of cartography. Abstracts of the $\mathrm{X}$ Jubilee International Scientific and Technical Conference "From imagery to map: digital photogrammetric technologies." Gaeta, Italy, 2010

[3] L.M.Brehovskih, Waves in sandwich mediums. - M.: Pub. AS USSR, 1956G. 